\title{
Frequency Shifts Due to the Interference of Resolved Peaks in Magnitude-Mode Fourier-Transform Ion Cyclotron Resonance Mass Spectra
}

\author{
Aleksey V. Tolmachev, Christophe D. Masselon, Gordon A. Anderson, \\ Harold R. Udseth, and Richard D. Smith \\ Environmental Molecular Science Laboratory, Pacific Northwest National Laboratory, Richland, \\ Washington, USA
}

\begin{abstract}
We have obtained relationships for frequency shifts resulting from the interference of spectral components for the magnitude mode Fourier transform. The approximation of a weak perturbation of well resolved peaks has been used. Both the low- and high-pressure limits for Fourier-transform ion cyclotron resonance (FTICR) operation have been considered. We have found that the shifts can be either negative or positive, depending on the initial phase and/or the choice of the time-domain interval. The magnitude of shifts generally does not exceed the peak width. In the approximation of small perturbations the shifts produced by multiple peaks are additive. We have compared theoretical results with experimental shifts for isotopic clusters of multiply charged insulin. Up to $1 \mathrm{ppm}$ frequency variations were experimentally observed for the insulin 5+ charge state, consistent with theoretical estimates. The peak interference is of particular significance in the case of bio-molecular mass spectra having a large number of peaks and covering a considerable dynamic range (i.e., relative abundance). We conclude that the common mass measurement procedure based on the location of the magnitude mode maxima of well resolved peaks can result in systematic mass measurement errors. The relationships obtained provide corrections for the frequency shifts and thus improve the mass measurement accuracy. (J Am Soc Mass Spectrom 2002, 13, 387-401) () 2002 American Society for Mass Spectrometry
\end{abstract}

$\mathrm{H}$ igh mass measurement accuracy is one of the key qualities of the Fourier-Transform Ion Cyclotron Resonance Mass Spectrometry (FTICR MS). This quality is particularly important for bio-molecular applications, where database search specificity is directly related to the mass measurement accuracy [1-5]. When complex mixtures of biological nature are studied, a single mass spectrum may carry information on hundreds of different compounds [6,7]. This situation applies when obtaining spectra for peptide or protein mixtures (e.g., using CE or LC separations in conjunction with the MS analysis of proteomes) [6-10], or in the monitoring biomolecule fragmentation [11-15]. Enhanced dynamic range is another quality highly desirable in such studies because of the greatly variable abundances of ion species of interest.

In this paper we consider interferences between peaks in FTICR mass spectra. We limit our consideration only to interferences resulting from the properties

Published online March 1, 2002

Address reprint requests to Dr. R. D. Smith, Environmetal Molecular Sciences Laboratory, Pacific Northwest National Laboratory, MSIN K8-98, P.O. Box 999, Richland, WA 99352, USA. E-mail: dick.smith@pnl.gov of the Fourier transform (FT), and do not consider factors associated with the physical interactions of ions, e.g., space charge [16-19], or phase-locking related interactions [20-23]. We consider the FT magnitude mode, which is commonly used for presenting mass spectra obtained using FTICR [24, 25]. Frequency shifts resulting from the mutual interferences of peaks are evaluated. We disregard the effects due to noise and consider the model case of a sum of ideal periodic (exponentially damped) signals. The aim is to characterize the limitations upon mass measurements for dense spectra and where peaks may cover a considerable dynamic range, and potentially provide a basis for the improved mass measurement accuracy.

Previous studies [26-29] have examined the effects of noise on the measurement precision. Phase correction may be employed for improving resolving power, using FT absorption spectra. This approach, referred to as phasing, has been studied for both FT NMR [30] and FTICR MS [31, 32]. Mass shifts induced by negative frequency peaks in linearly polarized FTICR signals have been considered [33]. The article reports that in the presence of ion molecule collisions the observed peak 
frequency can be shifted due to the overlapping with the wing of the negative-frequency peak. The extended mass calibration relation is proposed that exactly corrects for the shifts and improves mass calibration accuracy. Frequency shifts from partial overlap of positivefrequency peaks were also discussed.

Peak interference in the magnitude mode FTICR spectra was studied by Comisarow et al. [34-36]. It was shown that the magnitude-mode spectrum of overlapping spectral lines depends upon the relative phase of the corresponding time-domain signals [34]. The intensities, the valley height, and the apparent position of the two overlapping peaks were shown to depend upon the windowing function, damping constant, and the peak separation [35]. Frequency shifts of closely spaced peaks were systematically examined [36]. The study was based on the discrete Fourier transform applied to a model time-domain signal composed of two exponentially damped periodic components having equal amplitude. The frequency shifts were plotted as a function of the frequency separation between the two peaks, $\Delta f$, and the ratio of the acquisition time to the relaxation time, $T_{a} / \tau$. It was found that closely spaced peaks having $\Delta f<3 / T_{a}$ are shifted in frequency. The sign and magnitude of the shifts depend on the parameters $\Delta f$, $T_{a} / \tau$, the phase difference and the apodization function [37] used. It was problematic because of the many parameters involved to fully characterize the shifts and develop a correction methodology.

Here we use an alternative approach for the frequency shifts estimation, which is based on analytical relationships obtained using the continuous (or mathematical) Fourier transform applied to model time-domain signals. In this manner it is possible to characterize the frequency shift as a function of all essential parameters. Our consideration starts from the case of two components of equal intensity, same as considered in [36], and then embraces more general cases of two or more peaks having different intensities. Approximations used for obtaining the relationships include assumptions of a weak perturbation and well resolved peaks. We test the scope of applicability of the relationships obtained by comparison with direct calculations. The relationships allow one to predict conditions when the frequency shifts become significant. We carry out such an analysis and verify these results using experimental mass spectra, which show considerable shifts of well-resolved peaks.

\section{Methods}

The frequency shift calculation is based on a simple model time-domain signal consisting of a sum of only two components. We consider two classical cases. First, an infinite time-domain length and exponential damping with a constant characteristic time are assumed. This situation corresponds to the Lorentzian line shape [29]. This line shape may be assumed under high- pressure conditions, when the ICR time-domain signal damps considerably during the acquisition period. Next, we proceed with the opposite limit in which the damping time is assumed to be much larger then the acquisition period. In the frequency domain this situation results in peaks having the low-pressure line shape, described by the sinc function $[29,38]$. The frequency corresponding to a peak maximum is defined for both high- and low-pressure limits. This value is then compared with the corresponding exact frequency, and the frequency shift is determined. The calculations are based on approximate relationships (see Appendix) obtained from the mathematical definition of the Fourier transform. The results obtained for the doublet are then generalized for the case of a spectrum consisting of multiple peaks. The relationships for the frequency shifts are verified by exact, direct computations. Two approaches for the direct computations have been used. The first approach is based on the fast Fourier transform (FFT) of the model time-domain signal, consisting of a sum of exponentially damped periodic components. In order to precisely locate the peak position we implemented 0-filling and 3-point quadratic interpolation, as described in [36]. Another approach is based on the exact analytical relationships for the sine and cosine integral Fourier transforms [39] applied to the exponentially damped, phase-shifted sine wave. This approach allows one to calculate the peak shape with the doubleprecision accuracy of the computer, giving an accurate and reliable standard for testing approximate results.

Finally, we evaluate the theoretical results by comparison with actual FTICR mass spectra. The spectra were obtained using the 11.5 tesla FTICR mass spectrometer developed and constructed at Pacific Northwest National Laboratory. The instrument is controlled by an Odyssey (Finnigan, Bremen, Germany) data station, and equipped with an external electrospray ion source and an elongated cylindrical open-ended cell, described in details elsewhere [40]. The electrospray solvent consisted of $0.025 \%$ trifluoroacetic acid (TFA, Sigma Inc.); $0.1 \%$ acetic acid (Aldrich); the balance is water (nanopure 18.3 megaohm-cm resistivity). The bovine insulin (Sigma, St. Louis, MO) concentration used was $10 \mu \mathrm{g} / \mathrm{ml}$. A $+2 \mathrm{kV}$ voltage was applied to the ESI emitter, and charged species were injected through a 500 $\mu \mathrm{m}$ diameter heated metal capillary maintained at $160^{\circ} \mathrm{C}$. At the exit of the metal capillary, the ion beam was focused to the entrance of a quadrupole ion guide by an ion funnel interface [41-43]. The ions were accumulated in an external storage quadrupole before transfer to the FTICR cell. After trapping in the ICR cell, ions were excited using a broad range stored waveform inverse Fourier transform (SWIFT) [25] and detected at a 728 $\mathrm{kHz}$ acquisition frequency, for $512 \mathrm{~Kb}$ datapoints. Data were analyzed using software developed specifically for this work. 


\section{Results}

\section{Lorentzian Line Shape}

Let us first consider a simple case of a time-domain signal $s(t)$ consisting of two damped sinusoids, both having unit amplitude:

$$
\begin{aligned}
& s(t)=s_{0}(t)+s_{1}(t) \\
& s_{0}(t)=\sin \left(\omega_{0} t+\varphi_{0}\right) \exp (-t / \tau) \\
& s_{1}(t)=\sin \left(\omega_{1} t+\varphi_{1}\right) \exp (-t / \tau)
\end{aligned}
$$

Here $\omega_{0}$ and $\omega_{1}$ are angular frequencies, $\varphi_{0}$ and $\varphi_{1}$ are phases and $\tau$ is the exponential damping time constant. The frequency $\omega_{\mathrm{p}}$ of the power spectrum maximum for the first component can be shifted from the exact frequency $\omega_{0}$ due to a perturbation from the second component.

Consider a case where the two components are well resolved. The separation between the two components in terms of angular frequency can be defined as $\Delta \omega=$ $\omega_{1}-\omega_{0}$. For well-resolved peaks this separation must be larger than the half-width of the Lorentzian peak, $|\Delta \omega| \gg \Delta \omega_{1 / 2}$. Using the Lorentzian half-width [24], $\Delta \omega_{1 / 2}=2 \sqrt{3} / \tau$, we arrive at the following inequality:

$$
|\Delta \omega| \tau \gg 3.46
$$

The frequency shift $\delta \omega=\omega_{p}-\omega_{0}$ due to the perturbation from the second component can be estimated using relationships eq A5 and A8 (see Appendix). Unperturbed sine and cosine Fourier transforms $F_{s}(\omega)$ and $\mathrm{F}_{\mathrm{c}}(\omega)$ may be obtained by Fourier transform of the component $\mathrm{s}_{0}(\mathrm{t})$, eq 2 :

$$
\begin{aligned}
& F_{s}(\omega)=2 F_{L}^{2}(\omega)\left(\tau^{-1} \cos \varphi_{0}+\left(\omega-\omega_{0}\right) \sin \varphi_{0}\right) \\
& F_{c}(\omega)=2 F_{L}^{2}(\omega)\left(\tau^{-1} \sin \varphi_{0}-\left(\omega-\omega_{0}\right) \cos \varphi_{0}\right) \\
& F_{L}(\omega) \equiv \frac{\tau}{2 \sqrt{1+\left(\omega-\omega_{0}\right)^{2} \tau^{2}}}
\end{aligned}
$$

The Lorentzian function $F_{L}(\omega)[24,29]$ used here corresponds to the time-domain signal having unit amplitude. The relationships eq 5 and 6 are not exact, but very accurate in our case of a narrow peak, $\omega_{0} \gg 1 / \tau$, and for a frequency range close to the peak maximum, i.e., $\left|\omega-\omega_{0}\right| \sim \Delta \omega_{1 / 2}$. The power spectrum $\mathrm{P}(\omega)$, obtained from eq 5 and 6 using the definition eq A1, gives the Lorentzian line shape squared, $F_{L}^{2}(\omega)$. In order to estimate the frequency shift using eq A8, we need to find the second derivative of the unperturbed Lorentzian peak at its maximum, for $\omega=\omega_{0}$. Using eq 7 we obtain:

$$
\frac{d^{2} P}{d \omega^{2}}=-\frac{\tau^{4}}{2}
$$

The sine and cosine transforms of the small perturbation, $\delta F_{s}(\omega)$ and $\delta F_{c}(\omega)$, are similar to $F_{s}(\omega)$ and $F_{c}(\omega)$ above, but the second component $s_{1}(t)$ is used instead of $s_{0}(t)$. The condition of a narrow peak is replaced by the assumption that the separation between the two peaks is small compared to the unperturbed frequency, i.e., $|\Delta \omega| \ll \omega_{0}$. Having all four functions, $F_{s}(\omega), F_{c}(\omega), \delta F_{s}(\omega)$ and $\delta F_{c}(\omega)$, we can estimate the power spectrum of the perturbation $\delta P(\omega)$, using the relationship eq A5 from the Appendix:

$$
\delta P(\omega)=\frac{1}{2} \cos \Delta \varphi\left(\tau^{2} \frac{\omega-\omega_{0}}{\delta \omega_{1}}+\frac{1}{\delta \omega_{1}^{2}}\right)-\frac{\Delta \omega \tau}{2 \delta \omega_{1}^{2}} \sin \Delta \varphi
$$

Here $\Delta \varphi \equiv \varphi_{1}-\varphi_{0}$ is the initial phase difference, and $\delta \omega_{1} \equiv \omega-\omega_{1}$. The relationship eq 9 is an approximation valid for conditions of small perturbations, $\left|\omega-\omega_{0}\right|<$ $\tau^{-1}$, and well resolved peaks, eq 4 . For eq A8 we need the derivative of the perturbation, estimated for $\omega=\omega_{0}$. For our purpose of an approximate estimation we can ignore terms having $\delta \omega_{1}^{2}$, obtaining the following expression:

$$
\frac{d}{d \omega} \delta P\left(\omega=\omega_{0}\right) \approx-\frac{\tau^{2}}{2 \Delta \omega} \cos \Delta \varphi
$$

Substituting eq 8 and 10 into eq A 8 we arrive at the relationship for the frequency shift:

$$
\delta \omega=-\frac{1}{\Delta \omega \tau^{2}} \cos \Delta \varphi
$$

This relationship may be easily generalized for the case of two components having different amplitudes, $A_{0}$ and $A_{1}$. As shown in the Appendix, the frequency shift is a linear function of a perturbation in the limit of small perturbations considered. Thus, the frequency shift eq 10 needs to be corrected by the ratio of amplitudes $A_{r}=$ $A_{1} / A_{0}$ :

$$
\delta \omega=-\frac{A_{r}}{\Delta \omega \tau^{2}} \cos \Delta \varphi
$$

We see that the two exponentially damped periodic signals interact with each other so that the frequency $\omega_{p}$, defined as the location of the peak's maximum, deviates from the exact frequency $\omega_{0}$. Eq 12 has been obtained for the FT power spectrum $P(\omega)$, but it is also valid for the commonly used magnitude FT spectrum [29], because taking the square root of $P(\omega)$ does not change the location of the peak's maximum.

The perturbation arising from distant peaks de- 
creases inversely with the peak separation $\Delta \omega$, however distant peaks can still produce a significant perturbation, particularly when the intensity ratio $A_{r}$ is large (see following discussion for the case of large $\mathrm{A}_{r}$ ).

The frequency shift produced by several peaks may be estimated as a sum of shifts resulting from pair interferences, as obtained in the Appendix for weak perturbations. Thus, for a set of peaks each frequency shift can be found as $\delta \omega_{i}=\Sigma \delta \omega_{i j}$, where $\delta \omega_{i j}$ is the pair interference shift of the peak $i$ influenced by the peak $j$, estimated from eq 12.

To obtain an appreciation of the magnitude of frequency shifts we assume that the phase difference is zero, so that $\cos \Delta \varphi=1$. Consider a frequency domain FTICR mass spectrum having a peak at $\mathrm{m} / \mathrm{z} 1000$. The angular frequency corresponding to the peak's position is $\omega_{0} \sim 10^{6} \mathrm{~s}^{-1}$, for a $10 \mathrm{~T}$ magnetic field. Let us assume that a second peak is located at $\Delta \omega=1000 \mathrm{~s}^{-1}$, which corresponds to a mass difference of ca. $1 \mathrm{Da}$. In case of two isotopic peaks we may assume the intensity ratio $A_{r}$ $\sim 10$, i.e., corresponding to the case of a shift of the first isotopic peak caused by the perturbation from the monoisotopic peak. For the exponential damping time $\tau$ $=1 \mathrm{~s}$ we obtain from eq 12 the frequency shift $\delta \omega=$ $10^{-2} \mathrm{~s}^{-1}$, or $0.01 \mathrm{ppm}$ in terms of relative error. This shift is very small and may be disregarded in most practical cases. More significant shifts are produced for shorter transient lifetimes. For example for $\tau=0.1 \mathrm{~s}$ we obtain $\delta \omega=1 \mathrm{~s}^{-1}$, or a mass measurement error of 1 ppm. Finally, if we assume interference of ca. 10 peaks of same abundance, separated by $\Delta \omega \sim 100 \mathrm{~s}^{-1}$, we arrive at shifts of $\delta \omega \sim 10 \mathrm{~s}^{-1}$, or $10 \mathrm{ppm}$ (for $\tau=0.1 \mathrm{~s}$ ). The latter situation may occur in the case of a group of overlapping isotopic clusters or for highly multiply charged ions. We conclude that taking into account the frequency shifts of this origin is of significance for high accuracy FTICR mass measurements (for the mass measurement accuracy of ca. $1 \mathrm{ppm}$ or better) in many practical cases.

The above estimates were obtained for an absolute value of the frequency shift. When estimating the combined effect from several peaks, it is necessary to take into account the sign of each pair interference. Figure 1 shows the frequency shifts for a pair of Lorentzian peaks, for three different initial phase differences $\Delta \varphi$. The frequency shift eq 12 is negative for a positive $\Delta \omega$ in case of a zero initial phase difference. It follows that two peaks move apart as a result of interference when $\cos \Delta \varphi>0$, as the case in Figure 1a, for $\Delta \varphi=0$. If the two time-domain components are initially out-of-phase, then the frequency shift changes sign, and the peaks move closer (Figure $1 \mathrm{~b}, \Delta \varphi=\pi$ ). Finally, for a phase difference of $\pi / 2$ the interference is compensated and the peaks are positioned at frequencies corresponding to actual values. Parameters for the Figure 1 were chosen so as to make frequency shifts easily visible, and the approximation of resolved peaks (eq 4) is generally inadequate. The phase difference corresponding to the frequency shift compensation has
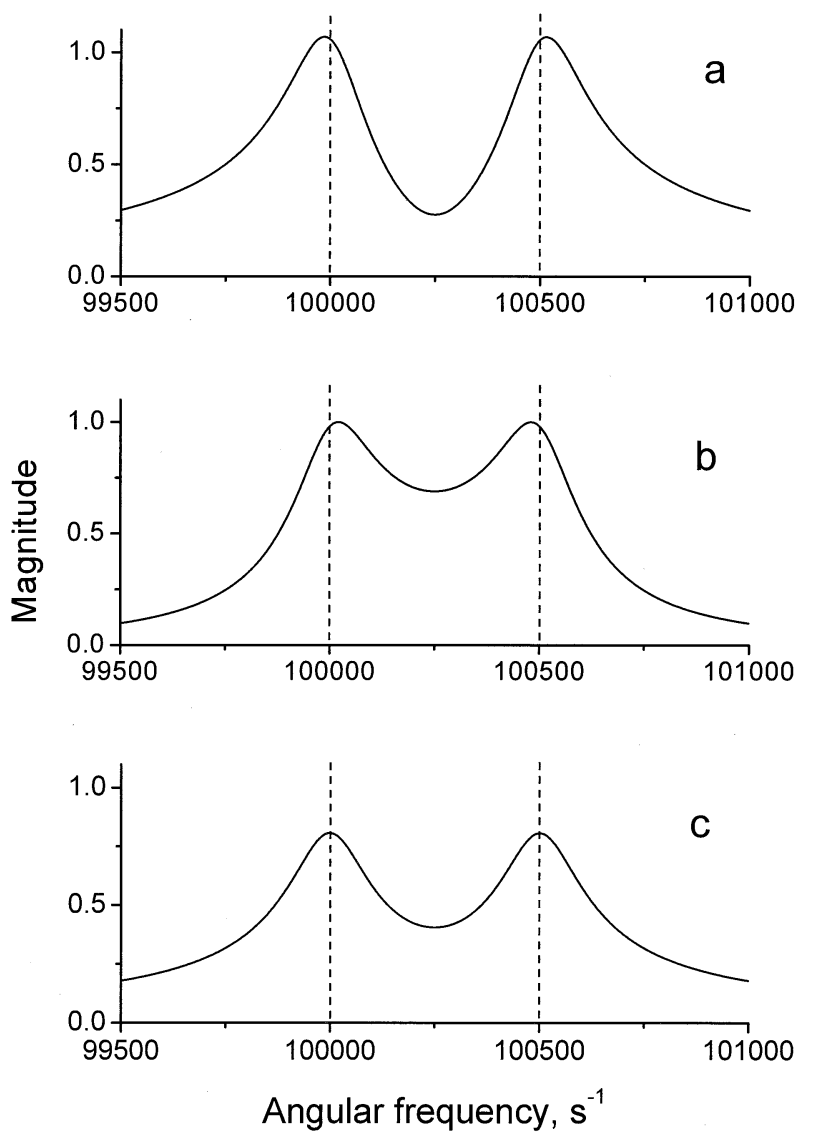

Figure 1. Magnitude FT of a sum of two periodical, exponentially damped signals, eq 1-3, having angular frequencies $\omega_{0}=1$ $\times 10^{5} \mathrm{~s}^{-1}$ and $\omega_{1}=1.005 \times 10^{5} \mathrm{~s}^{-1}$, and the exponential damping time $\tau=0.01 \mathrm{~s}$. The first component's phase is $\varphi_{0}=0$. The phase of the second component is (a) $\varphi_{1}=0$; (b) $\varphi_{1}=\pi$, and (c) $\varphi_{1}=$ $0.61 \pi$. Dashed lines show positions of exact, unperturbed frequencies. Magnitudes are normalized by the Lorentzian amplitude, $0.5 \tau$.

been adjusted empirically for this case to $0.61 \pi$, slightly different from the $\pi / 2$ value expected from the above approximate consideration.

The peak intensities in Figure 1 and the valley height between the peaks also exhibit the phase dependency. This observation is consistent with the study on the shape of two overlapping peaks in the magnitude FT spectrum [35]. It was found that the intensities, the valley height, and the apparent position of the two overlapping peaks are a function of the windowing function, damping constant, and the peak separation [35].

A practical consequence of the frequency shift dependence on phase is that the pair interference may be easily compensated by a slight adjustment of the time interval chosen for the Fourier transform. The phase difference $\Delta \varphi(t)$ of the two components changes with time $t$ as follows:

$$
\Delta \varphi(t)=\Delta \omega t+\varphi_{1}-\varphi_{0}
$$

In order to compensate for the peaks interference we 
can choose the starting time so that $\cos \Delta \varphi(t)=0$, or

$$
\Delta \varphi(t)=\pi(n+1 / 2), n=0,1, \ldots
$$

In practice this can be done by discarding a small initial interval of the time-domain signal, from 0 to $t_{0}$. The length $t_{0}$ of the skipped interval may be adjusted so as to minimize the frequency shift. Assuming that the time axis origin can be chosen arbitrarily, we can omit the initial phases in eq 13 and express the frequency shift as a function of $t_{0}$ :

$$
\delta \omega=-\frac{A_{r}}{\Delta \omega \tau^{2}} \cos \left(\Delta \omega t_{0}\right)
$$

i.e., the frequency shift eq 15 is a periodic function of the skipped time-domain interval $t_{0}$. It follows that $t_{0}$ value that provides the shift compensation may be chosen to be less then $\pi / \Delta \omega$. For example, for two interacting peaks separated by $\Delta \omega=100 \mathrm{~s}^{-1}$ (i.e., $\Delta f=16 \mathrm{~Hz}$ ) the initial interval to be discarded is not longer then $32 \mathrm{~ms}$, which is a minor fraction of a time-domain duration typical for FTICR measurements.

The relationship eq 15 suggests a possible approach for revealing frequency variations due to peak interferences in real data. This can be done by plotting a peak frequency as a function of the initial time-domain interval $t_{0}$ skipped prior to FT. In case of pair interference a simple cosine wave will be produced. The wave amplitude is $\delta \omega_{a}=A_{r} / \Delta \omega \tau^{2}$, and the period $T_{\Delta f}=1 / \Delta f$, where $\Delta f=\Delta \omega / 2 \pi$. This approach allows one to estimate measurement errors resulting from peak interference. The most accurate frequency value would be one corresponding to zeros of the cosine wave.

This method for frequency shift compensation can also be used to compensate for a net shift from a number of peaks, e.g., in the case of isotopic peak interference. In this case the function $\delta \omega\left(t_{0}\right)$ is a sum of cosines having amplitudes and periods defined by eq 15 for corresponding pair interferences. One can use the property of the eq 15 that it averages to zero over the interval $t_{0}$ equal to the period $T_{\Delta f}$. In case of several interfering peaks it is possible to average the shift over interval $t_{0}$ corresponding to the observed period of the frequency oscillations. An advantage of such an empirical approach is that it takes into account all interferences present in a mass spectrum under consideration. Generally the procedure will minimize the frequency shift only for one particular peak. However for some cases it may be possible to chose $t_{0}$ that minimizes the error for a group of peaks, as seen from an example for isotopic peaks considered below.

Derivation of the frequency shift relationship eq 12 involved several approximations. In order to evaluate the importance of these approximations we have performed direct calculations of the frequency shift and compared results with the relationship eq 12, see Figures 2 and 3 . The comparison shows that the approxi-

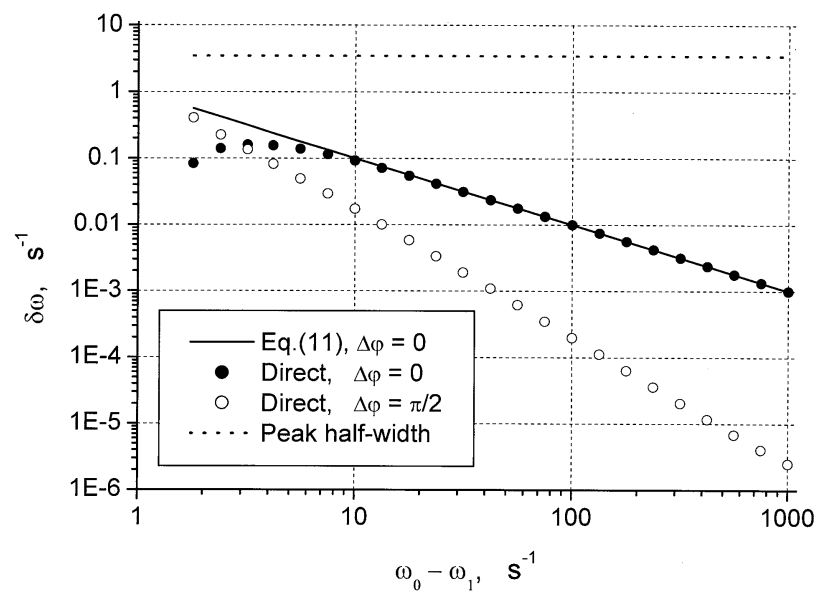

Figure 2. Angular frequency shift $\delta \omega$, produced by the interference from a closely spaced peak, versus frequency difference $\omega_{0}-$ $\omega_{1}$. The unperturbed angular frequency of the first peak is $\omega_{0}=10^{6}$ $\mathrm{s}^{-1}$; both components have same amplitudes: $A_{0}=A_{1}$. Highpressure limit: Exponential damping time $\tau=1 \mathrm{~s}$, infinite timedomain duration. Solid curve shows results from eq 12 , for $\Delta \varphi=$ 0 . Closed circles: $\Delta \varphi=0$, direct computation. Open circles: $\Delta \varphi=$ $\pi$, direct computation (absolute value of $\delta \omega$ is plotted). The peak half-width corresponding to the Lorentzian line shape [24] is shown by the horizontal dotted line.

mation gives reasonably good values for frequency shifts, but can deviate from the actual values when the assumption of a small perturbation breaks down. The frequency shift compensation based on the phase difference adjustment, eq 14, is quite efficient, as seen from direct computation results for $\Delta \varphi=\pi / 2$. In the case of a considerable perturbation the phase difference for exact compensation becomes different from $\pi / 2$, and

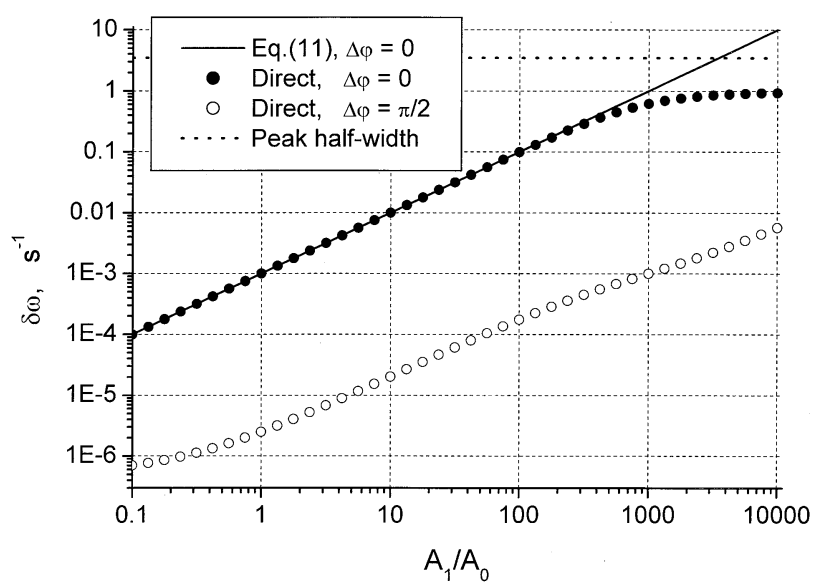

Figure 3. Angular frequency shift $\delta \omega$, produced by the interference from a closely spaced peak, versus peaks amplitude ratio $A_{1} / A_{0}$. The unperturbed angular frequency of the first peak is $\omega_{0}=10^{6} \mathrm{~s}^{-1}$; the frequency difference between the peaks is $\omega_{0}-\omega_{1}=1000 s^{-1}$. High-pressure limit: Exponential damping time $\tau=1 \mathrm{~s}$, infinite time-domain duration. Solid curve shows results from eq 12, for $\Delta \varphi=0$. Closed circles: $\Delta \varphi=0$, direct computation. Open circles: $\Delta \varphi=\pi$, direct computation (absolute value of $\delta \omega$ is plotted). The peak half-width corresponding to the Lorentzian line shape [24] is shown by the horizontal dotted line. 
some additional adjustment may be done, as in Figure 1c.

The unperturbed frequency used for Figures 2 and 3 (and also for Figures 5 and 6 below) is $\omega_{0}=10^{6} \mathrm{~s}^{-1}$, the same as used in the above estimates of frequency shifts for $1000 \mathrm{~m} / \mathrm{z}$ ions. Thus, the frequency shifts plotted along the vertical axis can be interpreted as relative shifts expressed in parts per million (ppm). The figures are plotted using the angular frequency presentation, $\omega=2 \pi f$, to simplify estimations using eq 12 .

The horizontal dotted line in Figures 2 and 3 shows the peak half-width, calculated for the Lorentzian line shape [24] as $\Delta \omega_{1 / 2}=2 \sqrt{3} / \tau$. The shifts calculated directly never exceeded the peak width. The magnitude of frequency shifts versus peak width is considered below (Discussion).

\section{Low-Pressure Line Shape}

The Lorentzian peak model considered above corresponds to the high-pressure limit in FTICR measurements, applicable when the damping time is much less than the time-domain acquisition period $T_{a}$ [29]:

$$
\tau \ll T_{a}
$$

In the opposite low-pressure limit, when $\tau \gg T_{a}$, both the peak width and the interference of peaks are defined by the total time-domain length $T_{a}$ rather then by $\tau$. Thus, we can disregard the exponential damping functions in eq 2 and 3 and assume the model timedomain signal of the following form:

$$
\begin{aligned}
& s(t)=s_{0}(t)+s_{1}(t), 0<t<T_{a} \\
& s_{0}(t)=\sin \left(\omega_{0} t+\varphi_{0}\right) \\
& s_{1}(t)=\sin \left(\omega_{1} t+\varphi_{1}\right)
\end{aligned}
$$

We follow the same steps as in the above calculations. Unperturbed sine and cosine Fourier transforms $F_{s}(\omega)$ and $F_{c}(\omega)$ have the following form:

$$
F_{s}(\omega)=\frac{1}{2\left(\omega_{0}-\omega\right)}\left(\sin \left(\left(\omega_{0}-\omega\right) \cdot T_{a}+\varphi_{0}\right)-\sin \varphi_{0}\right)
$$

$$
\begin{aligned}
F_{c}(\omega)= & -\frac{1}{2\left(\omega_{0}-\omega\right)}\left(\cos \left(\left(\omega_{0}-\omega\right) \cdot T_{a}+\varphi_{0}\right)\right. \\
& \left.-\cos \varphi_{0}\right)
\end{aligned}
$$

Here again we have used the approximation of a small variation of the angular frequency around the exact frequency, i.e., $\left|\omega_{0}-\omega\right| \ll \omega_{0}$, which has allowed us to omit in eq 20 and 21 terms corresponding to the negative frequency peak $[29,33]$. The line shape in the

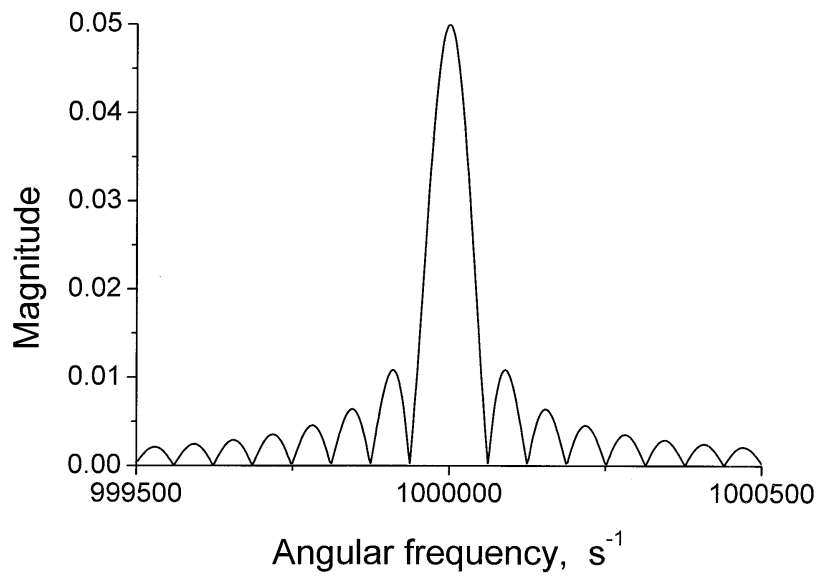

Figure 4. Low-pressure limit FTICR line shape [38] produced for a single periodic time-domain component, having angular frequency $\omega_{0}=10^{6} \mathrm{~s}^{-1}$. Time-domain acquisition period $T_{a}=0.1 \mathrm{~s}$.

FT power spectrum is as follows:

$$
P(\omega)=F_{L P}^{2} ; F_{L P}=\left|\frac{1}{\omega_{0}-\omega} \sin \left(\frac{\left(\omega_{0}-\omega\right) T_{a}}{2}\right)\right|
$$

Here $F_{L P}$ is the FTICR low-pressure line shape [38] shown in Figure 4. The second derivative at the peak maximum now is as follows:

$$
\frac{d^{2} P}{d \omega^{2}}=-\frac{T_{a}^{4}}{24}
$$

The small perturbation $\delta P$ from the second time-domain signal eq 19 can be found using approximation eq A5 (Appendix). The derivative of the perturbation is as follows:

$$
\frac{d}{d \omega} \delta P(\omega) \approx-\frac{T_{a}^{2}}{2 \Delta \omega} \cos \left(\Delta \varphi+\frac{\Delta \omega T_{a}}{2}\right) \cos \left(\frac{\Delta \omega T_{a}}{2}\right)
$$

Derivation of this relationship (not given) involved the approximation of well resolved peaks, which in the low-pressure limit corresponds to the following inequality [38]:

$$
|\Delta \omega| T_{a} \gg 7.58
$$

Another approximation used is that the separation between the two peaks is small compared to the unperturbed frequency, i.e., $|\Delta \omega| \ll \omega_{0}$.

Now we can combine eq 23 and 24 to obtain the frequency shift of the first peak, produced by the interference from the second peak:

$$
\delta \omega=-\frac{12 A_{r}}{\Delta \omega T_{a}^{2}} \cos \left(\Delta \varphi+\frac{\Delta \omega T_{a}}{2}\right) \cos \left(\frac{\Delta \omega T_{a}}{2}\right)
$$


We have included a coefficient taking into account the ratio of amplitudes of the time-domain components, $A_{r}=A_{1} / A_{0}$, following the same logic as was used for obtaining eq 12 .

Comparing the two frequency shifts, eq 12 and 26, we see that the absolute magnitude of shifts in both cases is inversely proportional to the frequency difference between the interacting components, and inversely proportional to the characteristic time squared. Both shifts depend on the initial phase difference $\Delta \varphi$. However, the shift in eq 26 has an additional periodic dependence on the time-domain length $T_{a}$. Thus, the shift eq 26 can be compensated by adjustment of the time interval $T_{a}$, so that:

$$
\frac{\Delta \omega T_{a}}{2}=\pi\left(\frac{1}{2}+n\right), n=0,1,2 \ldots
$$

Alternatively, one can compensate for the shift eq 26 by adjusting the initial phase difference $\Delta \varphi$. In practice this can be done by the phasing procedure, similar to one considered in the preceding section. We can replace the phase difference $\Delta \varphi$ in eq 26 by the time delay $t_{0}=$ $\Delta \varphi / \Delta \omega$, as follows:

$$
\delta \omega=-\frac{12 A_{r}}{\Delta \omega T_{a}^{2}} \cos \left[\Delta \omega\left(t_{0}+\frac{T_{a}}{2}\right)\right] \cos \left(\frac{\Delta \omega T_{a}}{2}\right)
$$

We can now set the delay time $t_{0}$ to one of the following values that provide the frequency shift compensation:

$$
t_{n}=\frac{1}{\Delta \omega} \pi\left(n+\frac{1}{2}\right)-\frac{T_{a}}{2}
$$

The frequency shift compensation efficiency is seen from exact calculations, Figures 5 and 6 . The compensation allows suppressing the shifts $>100$ times in the regions where the approximation of weak perturbation of two well-resolved peaks works well enough. Note that frequency difference values $\Delta \omega$ used for Figure 5 are chosen so that to set the second cosine in eq 28 to 1 , in order to eliminate $\Delta \omega$-dependent oscillations.

Comparison of the approximate relationship eq 28 and the direct computation results shows that the approximation is reasonably good, when two peaks are well resolved, and the perturbation is small enough for the linear approximation eq A5 to be applicable. The latter condition does not hold when the amplitude ratio is above $\sim 100$ for the parameters used for Figure 6. Note that the frequency shift magnitude for $A_{r}$ above 300 approaches the peak half-width $\Delta \omega_{1 / 2}=7.58 / T_{a}$ [38], shown by the horizontal dotted line. In such cases the approximations eq 26 and 28 are invalid, and more exact calculations should be used.

Direct computations used for Figures 2, 3, 5, and 6 are based on exact relationships for the Fourier transform applied to continuous signals eq 1 and 17. How-

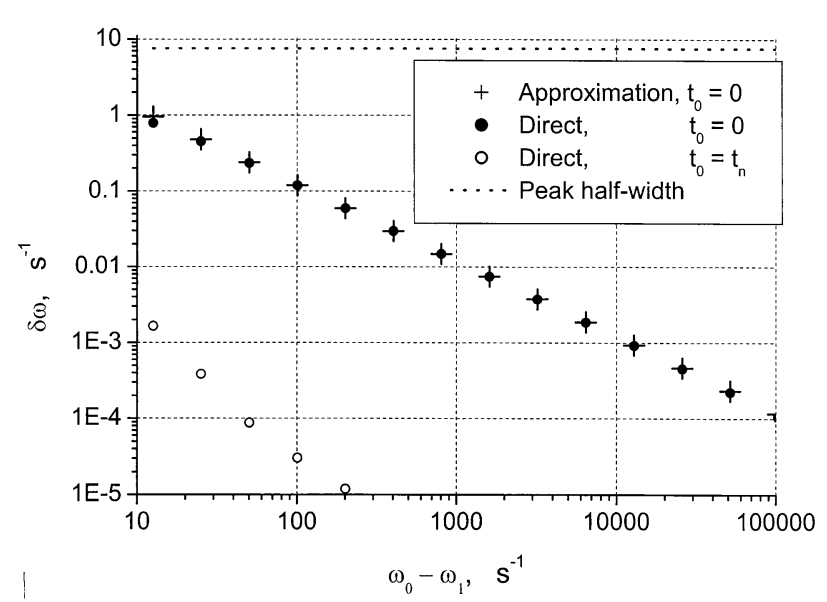

Figure 5. Angular frequency shift $\delta \omega$, produced by the interference from a closely spaced peak, versus frequency difference $\omega_{0}-$ $\omega_{1}$. The unperturbed angular frequency of the first peak is $\omega_{0}=10^{6}$ $\mathrm{s}^{-1}$; initial phase difference $\Delta \varphi=0$; both components have the same amplitude: $A_{0}=A_{1}$. Low-pressure limit: Time-domain length $T_{a}=1 \mathrm{~s}$, infinite exponential damping time. Crosses (+) show results from eq 28, for time delay $t_{0}=0$. Circles show direct computation results. Closed circles: $t_{0}=0$. Open circles: Time delay is set to frequency shift compensation, $t_{0}=t_{n}$, eq 29; the absolute value of $\delta \omega$ is plotted. The frequency differences plotted are: $\omega_{0}-\omega_{1}=4 \pi n / T_{a}, n=1,2,4, \ldots 8192$ (see text). The peak half-width corresponding to the low-pressure line shape [38] is shown by the horizontal dotted line.

ever, the same results can be obtained using the discrete FT procedure, e.g., fast Fourier transform, which is commonly used for processing FTICR spectra. We have tested both approaches and obtained equivalent results. However, under conditions of very weak interference

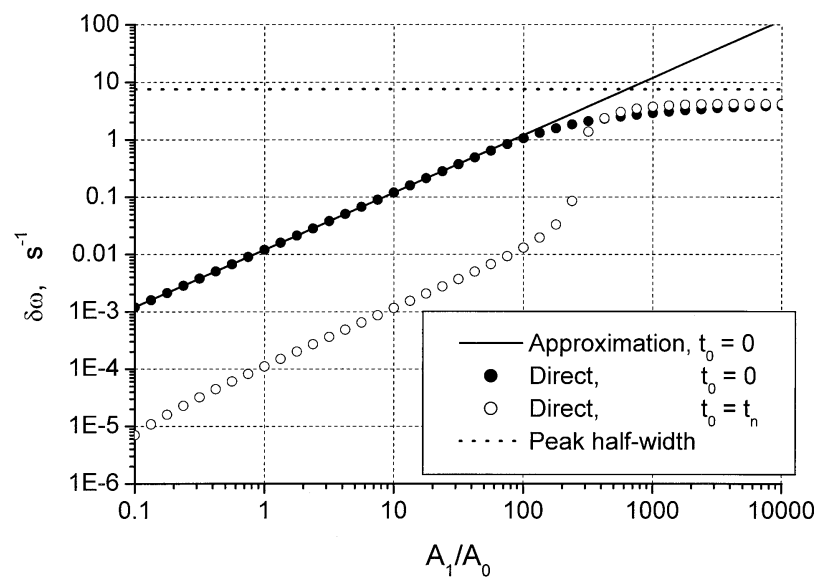

Figure 6. Angular frequency shift $\delta \omega$, produced by the interference from a closely spaced peak versus peaks amplitude ratio $A_{1} / A_{0}$. The unperturbed angular frequency of the first peak is $\omega_{0}=10^{6} \mathrm{~s}^{-1}$; initial phase difference $\Delta \varphi=0$; frequency difference between the peaks is $\omega_{0}-\omega_{1}=1000 \mathrm{~s}^{-1}$. Lowpressure limit: Time-domain length $T_{a}=1 \mathrm{~s}$, infinite exponential damping time. Solid curve shows results from eq 28, for time delay $t_{0}=0$. Circles show direct computation results. Closed circles: $t_{0}=0$. Open circles: Time delay is set to frequency shift compensation, $t_{0}=t_{n}$, eq 29; absolute value of $\delta \omega$ is plotted. The peak half-width corresponding to the lowpressure line shape [38] is shown by the horizontal dotted line. 
zero-filling procedure combined with interpolation must be used for accurate frequency determination using the discrete FT [36], which makes this approach more complicated for this kind of analysis.

Finally let us consider another form of the relationship for the frequency shift in the low-pressure case. We can rearrange eq 28 using the trigonometric identity $2 \cos \mathrm{A} \cos \mathrm{B}=\cos (\mathrm{A}+\mathrm{B})+\cos (\mathrm{A}-\mathrm{B})$, as follows:

$$
\delta \omega=-\frac{6 A_{r}}{\Delta \omega T_{a}^{2}}\left[\cos \left(\Delta \omega t_{0}\right)+\cos \left(\Delta \omega T_{\text {tot }}\right)\right]
$$

Here $T_{\text {tot }}=t_{0}+T_{a}$ is the total acquisition time, prior to truncating the time-domain by $t_{0}$ interval. The relationship eq 30 may be useful when analyzing data having a fixed total acquisition period. One can see that the frequency shift eq 30 includes a term $\cos \left(\Delta \omega \cdot T_{t o t}\right)$ that is independent of $t_{0}$ and is defined by the total acquisition length $T_{\text {tot }}$. Thus, frequency shifts in the low-pressure case generally can not be compensated by averaging over the period of a frequency variation $T_{\Delta f}$ as is the case for high-pressure shifts, eq 15 . However, this still can be done if the acquisition length is slightly adjusted, so that $\cos \left(\Delta \omega \cdot T_{\text {tot }}\right)=0$.

\section{Comparison with Experimental Results}

The above theoretical considerations were based on idealized concepts of FTICR operation in the high- and low-pressure limits. We also assumed that detected signals have ideal periodic exponentially damped form and are continuous. In contrast, real FTICR data have non-exponential, sometimes $\mathrm{m} / \mathrm{z}$-dependent damping, the acquisition process is discrete in time, and the detected signal is inharmonic. The real peak shape differs from high-pressure Lorentzian or low-pressure sinc shape, and can be affected by various factors [29, $44,45]$.

In order to test our theoretical results we have examined the real frequency shifts observed in an experimental mass spectrum. We have chosen a goodquality calibration mass spectrum from our $11.5 \mathrm{~T}$ FTICR instrument. The spectrum contains several charge states of insulin, which can be used for the DeCal calibration correction [46]. The acquisition period $T_{\text {tot }}=0.721 \mathrm{~s}$ has been used (Figure 7). Such a relatively short acquisition time is often used for high throughput measurements for bio-molecular applications, e.g., in LC/MS runs. The exponential damping time of the time-domain signal was estimated as $\tau \approx 2.1 \pm 0.5 \mathrm{~s}$, which is larger then the acquisition period. Formally this situation is between the low and high-pressure cases. To treat this case we have first directly calculated the frequency shifts, as done above for verifying our approximations. Then we also have applied the approximation obtained above to test its usefulness for treating experimental data.

The frequency-domain interval with $5+$ insulin iso-

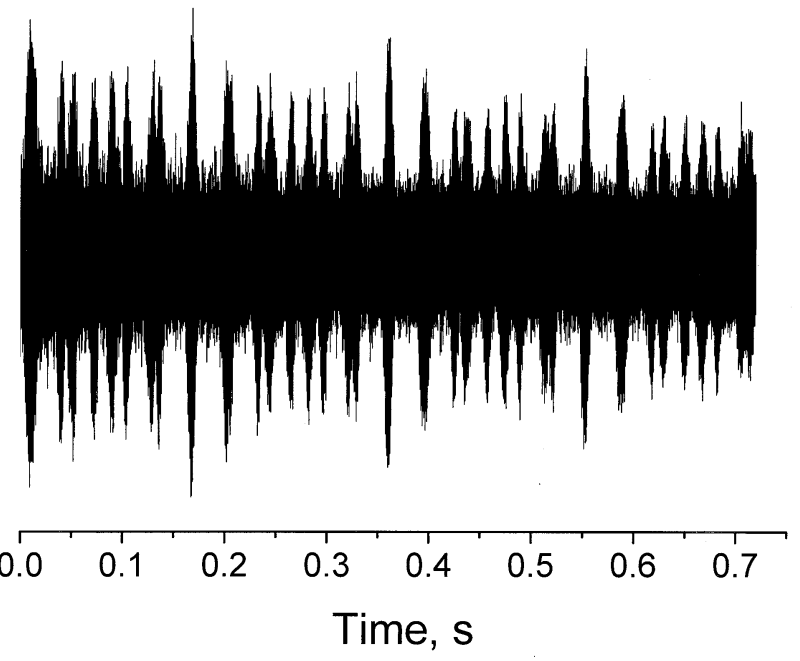

Figure 7. Experimental FTICR time-domain signal, obtained for a calibration mixture containing multiply charged ions of insulin. The acquisition period is $0.721 s ; 524288$ data points are used. The damping constant estimated based on the damping rate of the transient is $\tau \approx 2.1 \pm 0.5 \mathrm{~s}$.

topic cluster is shown in Figure 8a. Magnitude mode presentation of the Fast Fourier transform, with no zero filling, is used. To reveal details of the experimental line shape we have zoomed the main peak no. 5, Figure $8 b$. Here we have implemented zero filling of 3rd order, i.e., the experimental transient length has been increased 8 times by adding zeros prior to FFT. The peak has half-width $\Delta f_{1 / 2} \approx 1.7 \mathrm{~Hz}$, corresponding to a resolving power $\sim 90 \mathrm{~K}$. This half-width is consistent with that expected for the low-pressure line shape [38]:

$$
\Delta f_{1 / 2}=\frac{3.79}{\pi T_{a}}=1.68 \mathrm{~s}^{-1}
$$

Figures 9 and 10 show frequencies of the isotopic peaks versus the initial time interval $t_{0}$ discarded prior to $F T$. The elimination of the initial part of a time-domain is equivalent to an additional time delay, so the parameter $t_{0}$ is referred to as the time delay in following considerations. The frequency shift $\delta f, \mathrm{~Hz}$, of the magnitude peak relative to the unperturbed frequency is shown. The preceding theoretical treatment used angular frequencies $\omega=2 \pi f$, so the factor $2 \pi$ must be taken into account when comparing shifts in Figures 9, 10, and 11 with the above results.

Figure 9 shows experimental results obtained for the spectrum from Figure 8a. All curves start at $\delta f=0$ because the frequencies for the whole time-domain (for $\left.t_{0}=0\right)$ are taken as the reference, or unperturbed, frequencies. Next, Figure 10 shows the frequency shifts calculated for the model isotopic cluster of insulin 5+, having parameters listed in Table 1; the time-domain period $T_{\text {tot }}=0.721 \mathrm{~s}$ has been used, corresponding to the experimental value. The exponential damping time used for calculations is taken from the experimental transient shown in Figure 7, $\tau=2.1 \mathrm{~s}$. Zero initial phases 

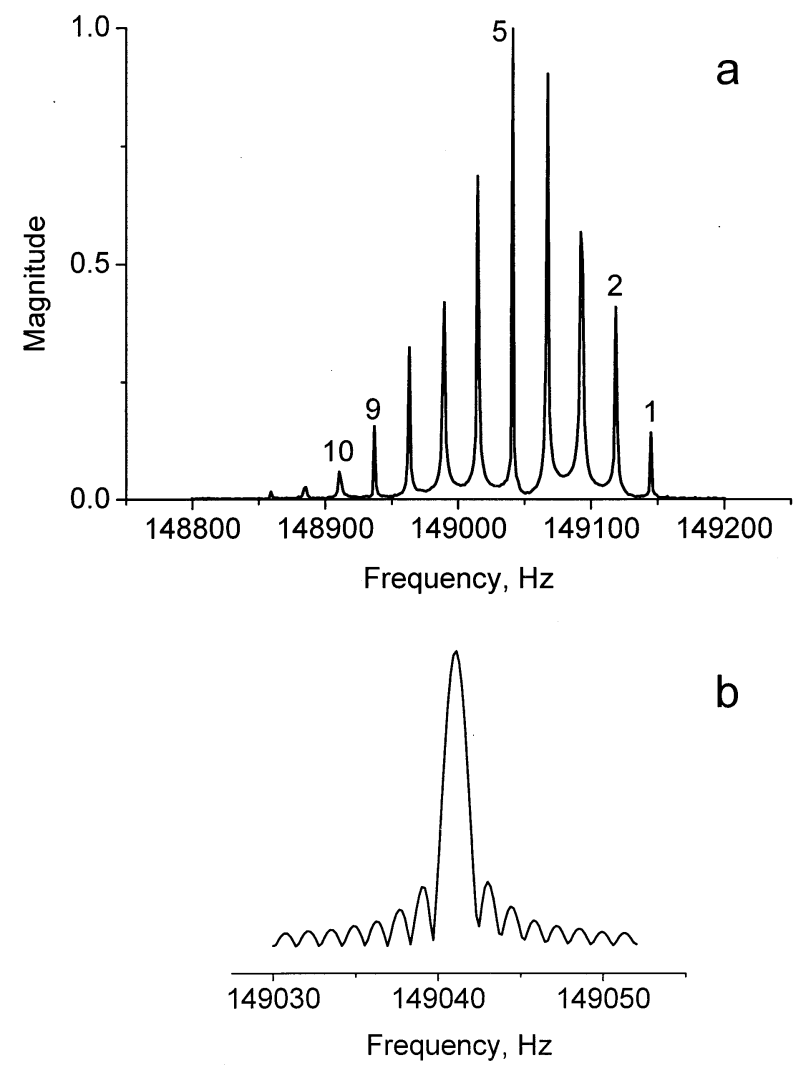

Figure 8. Portions of the experimental mass spectrum showing insulin $5+$ ion, in the frequency-domain magnitude mode. Amplitude is normalized by the most abundant isotopic peak intensity. (a) Isotopic cluster of the insulin 5+ ion; no zero filling is applied. Peaks marked by numbers are used for the analysis of frequency shifts shown in Figures 9-11. (b) Experimental peak line shape is shown for the isotopic peak no. 5. Zero filling of the third order is used, corresponding to the transient duration increased by factor of 8 .

have been assumed for all 10 components. Each frequency shift has been calculated as a sum of 9 pair interferences. An exact frequency of each corresponding component has been used as unperturbed reference frequency.

The amplitude of frequency variations in Figures 9 and 10 reaches $0.2 \mathrm{~Hz}$, or $\sim 1.3 \mathrm{ppm}$ for smaller isotopic peaks on both sides of the isotopic cluster, peaks no. 1 and 10 . The frequency beats repeat with period $T_{b}=38$ ms. This quantity corresponds to a period of the timedependent phase eq 13:

$$
T_{\Delta f}=1 / \Delta f
$$

Here $\Delta f=\Delta \omega / 2 \pi$ is the frequency interval between isotopic peaks, $\Delta f=26 \mathrm{~Hz}$, see Figure $8 \mathrm{a}$. Frequencies of the higher-frequency (lower $\mathrm{m} / \mathrm{z}$ ) peaks tend to increase during the beats, while lower-frequency peaks of the envelope have negative shifts. In the mass scale this corresponds to mass intervals increased on average. Thus, calculation of the 0-charge molecular weight corresponding to the isotopic cluster will result in

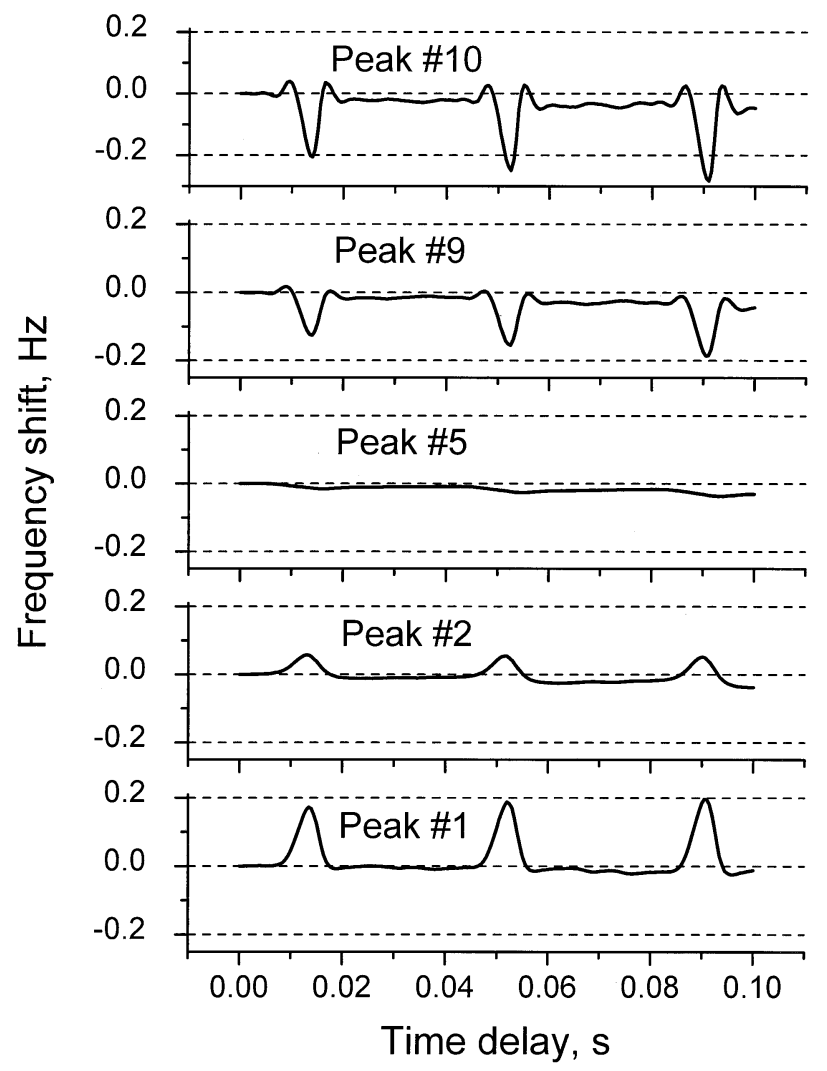

Figure 9. Frequency shifts versus delay time for peaks of the insulin 5+ isotopic cluster from the experimental spectrum shown in Figure 8(a). The delay time is defined as the length of initial time-domain interval being rejected prior to FFT, see text. Peaks chosen for the frequency shift analysis are no. 1,2, 5, 9, and 10, as labeled in the Figure 8(a).

systematic mass error, when the frequencies are measured for the time-domain segment corresponding to the frequency beats. In theory for zero initial phase difference, if the full time-domain is used for FT $\left(t_{0}=0\right)$, then the frequency shifts are maximized, as in Figure 10 for $t_{0}=0$. However in real measurements the beginning of the time-domain signal does not coincide with the moment of in-phase motion of all ions. One reason for this is that the experimental detection of the timedomain signal typically starts at some time delay after the end of excitation. Additionally, excitation of ions generally results in non-zero phase differences, e.g., SWIFT excitation used in our experiments. The mass spectrum has been acquired using $41 \mathrm{~ms}$ long SWIFT excitation sequence followed by $1 \mathrm{~ms}$ delay before starting data acquisition. This explains why experimental frequency shifts in Figure 9 are displaced along $t_{0}$ axes, relative to theoretical curves in Figure 10.

Comparing the experimental and theoretical frequency shifts in Figures 9 and 10 we can see that both the amplitudes of beats and the time interval between the beats are similar. We conclude that the main origin of the experimental frequency variations is due to the interference of the FT components, as considered above.

The direct computation of frequency shifts in Figure 


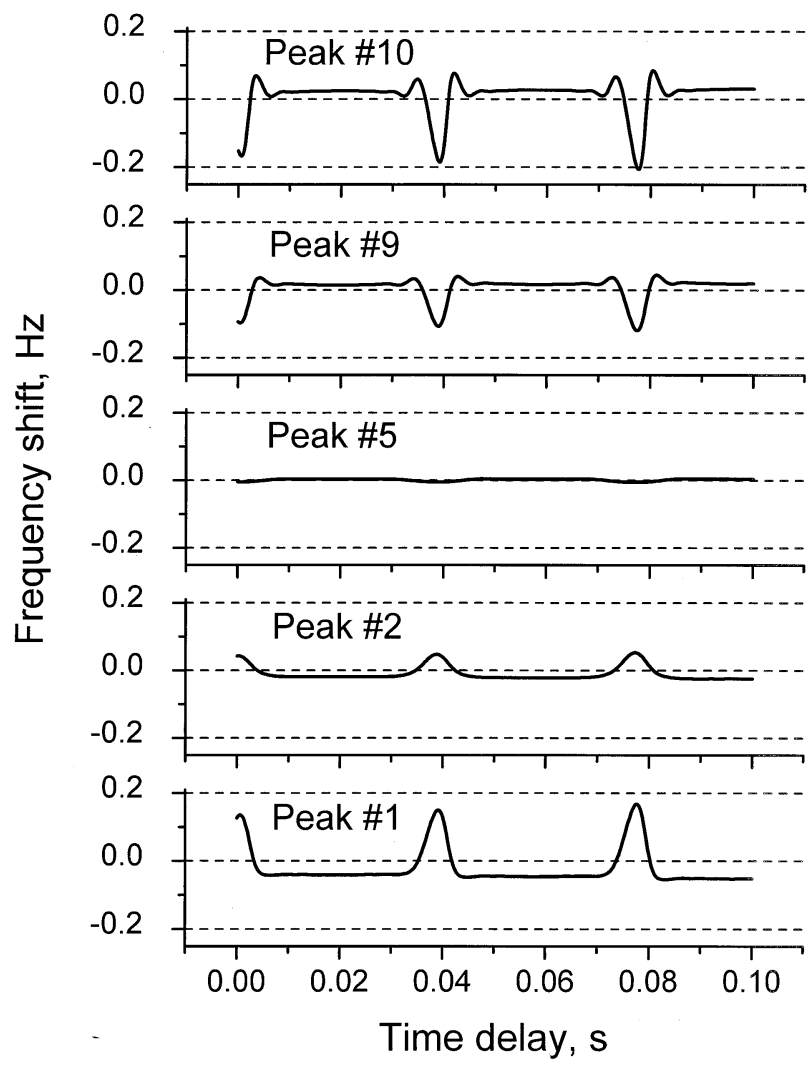

Figure 10. Frequency shifts versus delay time for peaks of the insulin 5+ isotopic envelope, calculated for theoretical abundances of the insulin isotopic peaks, using the total time-domain interval of $0.721 \mathrm{~s}$ and the exponential damping time $\tau=2.1 \mathrm{~s}$, same as in the experimental spectrum in Figure 8(a). Parameters of the model isotopic cluster used for the calculation are listed in Table 1; a zero initial phase has been assumed for all 10 peaks. The frequency shift has been calculated as a sum of 9 pair interferences. The same isotopic peaks (no. 1, 2, 5, 9, and 10) were chosen as in Figure 9.

10 took into account both the exponential damping and the actual length of the acquisition time, avoiding the approximations used in the above high- and lowpressure treatments. The three beats over the time interval of $0.1 s$ have increasing amplitudes due to decreasing time-domain length remaining after rejection of a certain time delay $t_{0}$ :

$$
T_{a}=T_{\text {tot }}-t_{0}
$$

An alternative way to obtain the dependence $\delta f\left(t_{0}\right)$ would be to move both the beginning and the end of the interval taken for $F T$, so that the value $T_{a}$ is constant, independent of $t_{0}$, i.e., in terms of windowing [36, 37], apply the rectangular window $R(t)=1$ for $t_{0}<t<t_{0}+$ $T_{a}$. In this case it would be easier to apply the approximate relationship eq 28 to analyze the shifts. Adjusting $t_{0}$ and $T_{a}$ in eq 28 is a possible way for minimization of the frequency error. This approach can be applied for experimental data obtained under the low-pressure conditions. We leave such an analysis, together with various approaches for frequency shift corrections, for

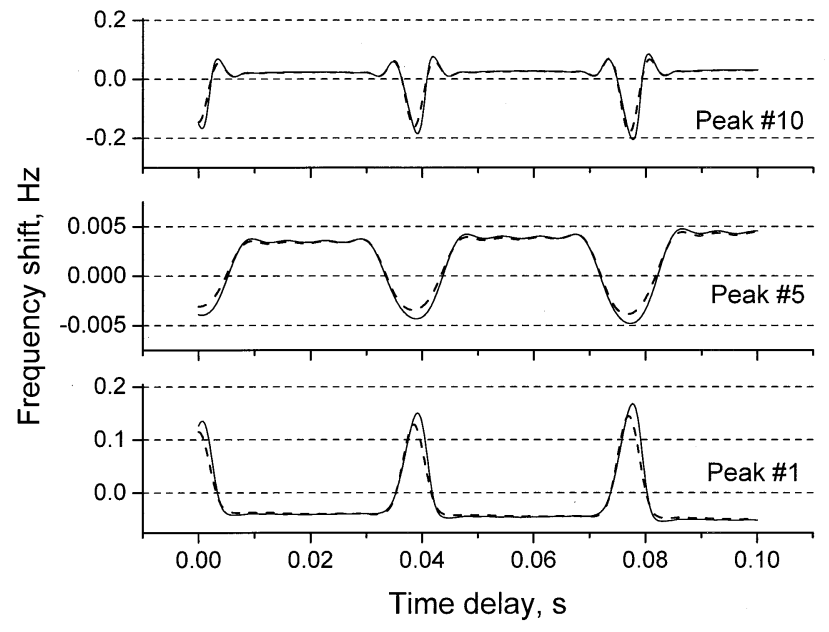

Figure 11. Frequency shifts versus delay time, calculated for the model isotopic cluster of insulin $5+$ ion, for peaks 1,5 , and 10 . The solid curve corresponds to the direct calculations, same as shown in Figure 10. The dashed line shows results obtained using the approximate relationship for the low-pressure limit, eq 28, which disregards the transient damping over the acquisition period. Sum of frequency shifts resulting from 9 pair interferences is taken. Parameters of the model isotopic cluster used for the calculation are listed in Table 1; a zero initial phase has been assumed for all 10 peaks.

future studies.

Experimental conditions considered here are quite close to the low pressure limit, when one can neglect the signal damping over the acquisition period, as seen from the experimental transient in Figure 7. This allows us to use the relationship eq 28 for a simple approximate estimation of the frequency shifts. Results of such estimation are shown in Figure 11 (dashed curve), together with the direct calculations discussed above (solid curve). The frequency shift for each peak is estimated as $\delta \omega_{i}=\Sigma \delta \omega_{i j}$, where $\delta \omega_{i j}$ is the frequency shift obtained using eq 28 for the shift of the peak $i$ resulting from interference with the peak $j$, for $T_{\text {tot }}=$ $0.721 \mathrm{~s}$ and peak parameters listed in Table 1; all 9 pair interferences are summed. The approximate calculations do not differ much from the accurate results, meaning that the low pressure approximation can be used for this case which has a low ratio of the acquisi-

Table 1. Parameters of the model isotopic cluster of insulin 5+ used for frequency shifts estimations

\begin{tabular}{lccc}
\hline Peak no. & Mass, Da & Abundance & Frequency, Hz \\
\hline \hline 1 & 1145.92 & .028 & 149069.9583 \\
2 & 1146.12 & .089 & 149043.9453 \\
3 & 1146.32 & .153 & 149017.9414 \\
4 & 1146.52 & .186 & 148991.9466 \\
5 & 1146.72 & .178 & 148965.9609 \\
6 & 1146.92 & .143 & 148939.9842 \\
7 & 1147.12 & .099 & 148914.0165 \\
8 & 1147.32 & .061 & 148888.0579 \\
9 & 1147.52 & .033 & 148862.1084 \\
10 & 1147.72 & .017 & 148836.1679 \\
\hline
\end{tabular}


tion time to the damping constant, $T_{a} / \tau=0.34$. Other characteristics of the low-pressure regime have already been mentioned above, namely, peak widths corresponding to the low-pressure relationship eq 31 and the experimental line shapes (Figure $8 \mathrm{~b}$ ) being similar to the theoretical low-pressure line shape, Figure 4.

The experimental frequency shifts in Figure 9 show a small negative overall trend that is not present in the theoretical plots in Figures 10 and 11, and that may be due to physical factors omitted in our analysis. Slow frequency drifts observed using FT over consecutive time-domain segments have been studied in [47]. The drifts have been attributed to evolution of the shape of the coherently orbiting ion packets; a frequency drift compensation procedure has been suggested that considerably improves the resolving power [20, 47].

Here we have implemented an alternative method for the frequency drifts visualization based on plotting the peak frequency versus the length of the initial time interval rejected prior to FT. The approach is similar to the segmented FT [47], but can be used to monitor faster frequency variations having characteristic times shorter than the length of the moving time interval $T_{a}$. It allows one to filter out the frequency variations due to peak interference, as considered above. The approach can be applied to practical, relatively short time-domain data without sacrificing the accuracy of frequency determination, because instead of consecutive segmenting, a moving time-domain interval can be used that is just slightly shorter than the total time-domain duration. Preliminary results obtained using this approach for sample bio-molecular spectra show that the physical frequency shifts often dominate over the shifts due to FT peak interference. Further improvement in the FTICR mass measurement accuracy will require consideration of frequency shifts of both physical and mathematical origin, which will be subject of a future work.

\section{Discussion}

The frequency shifts due to a short transient life time $\tau$, or insufficient acquisition time $T_{a}$, can be suppressed by adjusting experimental/measurement conditions to increase both time periods. We can use relationships eq 12 and 28 to estimate the amplitude of the shifts $\delta \omega_{a}$ defined as the absolute value of $\delta \omega$, eq 12 and 28, taken without the cosine functions. The value $\delta \omega_{a}$ is inversely proportional to the square of the effective duration of a transient $\mathrm{T}_{\text {eff: }}$ :

$$
\delta \omega_{a} \propto \frac{1}{|\Delta \omega| T_{\mathrm{eff}}^{2}}
$$

Here we have assumed $T_{\text {eff }}=\tau$ for the high pressure case, eq 11, and $T_{\text {eff }}=T_{a}$ for the low pressure limit, eq 28 . Both the relaxation time $\tau$ and the acquisition period $T_{a}$ are related to the total FTICR measurement cycle time. We conclude that optimal parameters would represent a compromise between the accuracy and the throughput of measurements.

The inverse relative error resulting from the frequency shift can be defined as $R_{\omega}=\omega / \delta \omega_{a}$. This quantity is similar to the relative mass resolving power $R_{m}=m / \Delta m$. Classical relationships for the FTICR resolving power in the low and high-pressure limits are as follows [24]:

$$
\begin{aligned}
R_{m}= & \frac{q B \tau}{2 \sqrt{3} m} \\
& - \text { high-pressure limit, Lorentzian peaks } \\
R_{m}= & \frac{0.132 q B T_{a}}{m}-\text { low pressure, sinc line shape }
\end{aligned}
$$

Here $m$ is ion mass, $q=z e$ is ion charge, $e$ is elementary charge, $B$ is magnetic field. The mass resolving power can be expressed in frequency terms as $R_{m}=\omega / \Delta \omega_{1 / 2}$ [24], and for the relative frequency shift we obtain:

$$
R_{\omega}=R_{m} \frac{\Delta \omega_{1 / 2}}{\delta \omega_{a}}
$$

Substituting for the peak half-width $\Delta \omega_{1 / 2}$ corresponding expressions for high- and low-pressure limits [24], we arrive at the following relationships:

$$
\begin{aligned}
R_{\omega}= & R_{m} \frac{2 \sqrt{3}|\Delta \omega| \tau}{A_{r}} \\
& - \text { high-pressure limit, Lorentzian peaks }
\end{aligned}
$$

$$
\begin{aligned}
R_{\omega}= & R_{m} \frac{0.632|\Delta \omega| T_{a}}{A_{r}} \\
& - \text { low pressure, sinc line shape }
\end{aligned}
$$

Thus, the inverse frequency shifts are proportional to the resolving power, multiplied by the characteristic time, and we can use the well-known relationships for the theoretical limit of $R_{m}$ to estimate $R_{\omega}$. Since $R_{m}$ is proportional to the characteristic time, eq 35 and 36, we conclude that the frequency shifts will be reduced with increasing mass resolving power, proportionally with $R_{m}^{2}$.

For the case of well resolved peaks considered here we can use inequalities eq 4 and 25 , arriving at the following upper limits for the relative frequency shift:

$$
R_{\omega} \gg R_{m} \frac{12}{A_{r}}
$$

- high-pressure limit, Lorentzian peaks 


$$
R_{\omega} \gg R_{m} \frac{4.8}{A_{r}}-\text { low pressure, sinc line shape }
$$

We see that the inverse relative frequency shifts $R_{\omega}$ are much greater than the mass resolving power $R_{m}$, provided that the amplitude ratio $A_{r}$ is not large, $A_{r} \sim 1$. In terms of absolute frequency shifts this implies that the shift is small compared to the peak width.

Relationships eq 38-41 can be used only when the approximation of a weak perturbation applies. The approximation has been tested in comparisons of the approximate relationships and direct calculations (Figures $2,3,5$, and 6 ). Let us consider the plots $\delta \omega$ versus $A_{r}$ shown in Figures 3 and 6 , for $\omega_{0}=10^{6} s^{-1}$ and $\Delta \omega=$ $-1000 s^{-1}$. We see that for the amplitude ratio $A_{r}$ larger than $\sim 200$ the approximations eq 12 and 28 deviate from accurate values, although the peaks are well resolved. Thus the approximations can be inaccurate in case of distant peaks if the intensity ratio is too high. The limit for possible frequency shifts can be estimated from direct calculations as follows. The relative peak width is 3.5 ppm for the high pressure case, Figures 2 and $3\left(\omega_{0}=10^{6} \mathrm{~s}^{-1}\right.$ and $\left.\tau=1 \mathrm{~s}\right)$. For the low pressure case, Figures 5 and 6 , the relative peak width is $7.6 \mathrm{ppm}$ $\left(\omega_{0}=10^{6} \mathrm{~s}^{-1}\right.$ and $\left.\mathrm{T}_{a}=1 \mathrm{~s}\right)$. The peak half-width is shown by dotted line in all four figures. We can see that the directly computed frequency shifts level at values not exceeding the peak width, or in terms of the inverse relative quantities:

$$
R_{\omega}>R_{m}
$$

This limit for the frequency shifts follows from exact calculations (no approximations). The condition sets the limit to both the frequency shifts and to the scope of the approximate relationships obtained.

Finally, we can express the inverse relative shifts in terms of physical parameters, such as ion $\mathrm{m} / \mathrm{z}$ and magnetic field $B$. Using the unperturbed cyclotron frequency $q B / m$ for $\omega$, we obtain:

$$
\begin{aligned}
R_{\omega}= & \frac{e^{2} B^{2} \tau^{2} \Delta(m / z)}{A_{r}(m / z)^{3}} \\
& - \text { high-pressure limit, Lorentzian peaks } \\
R_{\omega}= & \frac{e^{2} B^{2} T_{a}^{2} \Delta(m / z)}{12 A_{r}(m / z)^{3}}-\text { low pressure, sinc line shape }
\end{aligned}
$$

Here $\Delta(m / z)$ stands for $m / z$-separation of the two interacting peaks. The frequency shifts increase proportionally to the cubic power of $m / z$, which makes the corrections of particular importance for high $m / z$ ions. Equation 43 and 44 also show that frequency shifts improve with increasing magnetic field, $\delta \omega_{a} / \omega \propto 1 / B^{2}$, which implies one more advantage of the high magnetic field FTICR MS [25].

We did not consider here the use of apodization [37] and its effect on the frequency shifts resulting from interference of peaks. This problem was studied in [36] using direct frequency shift calculations for model doublets of exponentially damped signals. It was found that maximum absolute shifts observed for apodized signals are larger than for unapodized signals, although for certain conditions apodization can help in the shift compensation. A similar conclusion can be deduced from our results if one considers that elimination of an initial time interval used above is equivalent to applying the rectangular window $R(t)$, as defined in [36, 37]. Thus obtained above functions $\delta \omega\left(t_{0}, \mathrm{~T}_{a}\right)$ can be interpreted as shift dependence versus the rectangular apodization parameters. Also, the exponentially damped signal eq 1 can be expressed via non-damped one, eq 17, multiplied by the exponential window function $W(t)=$ $\exp (-t / \tau)$. It follows that the high-pressure results can be considered as frequency shifts for long undamped signals apodized using the exponential window function.

Generally, apodization can increase the effective transient duration by cost of increasing the relative input of parts of the transient that have low intensity and, consequently, low signal-to-noise ratios [29]. In this sense apodization cannot increase the informational content of FTICR data [29]. Thus, the efficiency of apodization in terms of frequency shifts compensation must be considered taking into account noise present in the signal.

\section{Conclusion}

We have obtained relationships for frequency shifts resulting from the interference of spectral components for magnitude mode Fourier transforms. The shifts can have both negative and positive sign, depending on the initial phase and/or the choice of the time-domain interval. In the approximation of small perturbations the shifts produced by multiple peaks are additive. The magnitude of shifts generally does not exceed the peak width.

Theoretical results have been compared with experimental shifts obtained for a high quality mass spectrum for multiply charged insulin having a well resolved isotopic structure. In this case the frequency shifts are very pronounced because of a combined interference of closely positioned isotopic peaks. We have observed up to $1 \mathrm{ppm}$ frequency variations in the real spectrum of the insulin $5+$ charge state, which agrees well with theoretical estimates.

The peak interference sets the fundamental limit for the mass measurement accuracy attainable in FTICR MS using the common approach (i.e., based on the location of the magnitude mode peak maxima). In other words, for ideal mass measurement conditions there can still be some systematic inaccuracies. The relationships obtained allow one to correct for these frequency 
shifts and to further improve the mass measurement accuracy.

Inaccuracies due to peak interference may be of particular significance in the case of bio-molecular mass spectra that have a large number of peaks, covering a considerable dynamic range. Possible approaches for shift corrections in such cases will be the subject of subsequent studies.

An immediate consequence of our findings is that the peak interference must be taken into account in cases when the high mass measurement accuracy is essential, e.g., when choosing peaks for calibration or for defining mass values for a database search that requires a high mass measurement accuracy. In such cases any closely positioned, high intensity peaks will degrade the achievable accuracy.

\section{Appendix}

\section{Peak Frequency Shift Due to a Perturbation in the FT Power Spectrum}

The FT power spectrum $P(\omega)$ may be obtained from sine and cosine Fourier transforms, $F_{s}(\omega)$ and $F_{c}(\omega)$ [39], as follows:

$$
P(\omega)=F_{s}(\omega)^{2}+F_{c}(\omega)^{2}
$$

Let us consider a frequency interval around a maximum of a power spectrum peak. The maximum position $\omega_{p}$ may be obtained from the following condition of the extremum:

$$
\frac{d}{d \omega} P(\omega)=0 \text { for } \omega=\omega_{p}
$$

In the case of unperturbed single component signal the position of the maximum $\omega_{p}$ coincides with the frequency of the time-domain component $\omega_{0}$. Let us introduce a small perturbation, resulting from a presence of additional components in the time-domain signal. Due to linearity of the sine and cosine transforms we may express the perturbed power spectrum $P_{1}(\omega)$ as follows:

$$
P_{1}(\omega)=\left(F_{s}(\omega)+\delta F_{s}(\omega)\right)^{2}+\left(F_{c}(\omega)+\delta F_{c}(\omega)\right)^{2}
$$

Here $\delta F_{s}(\omega)$ and $\delta F_{c}(\omega)$ are sine and cosine Fourier transforms of the small perturbation. The perturbed spectrum may be expressed through the unperturbed one:

$$
\begin{aligned}
& P_{1}(\omega)=P(\omega)+\delta P(\omega) \\
& \delta P(\omega) \approx 2 F_{s}(\omega) \delta F_{s}(\omega)+2 F_{c}(\omega) \delta F_{c}(\omega)
\end{aligned}
$$

We have omitted two small second order terms in the right-hand side, $\delta F_{s}(\omega)^{2}$ and $\delta F_{c}(\omega)^{2}$. The location of the perturbed peak maximum can be found from the following extremum condition:

$$
\frac{d}{d \omega} P=-\frac{d}{d \omega} \delta P \text { for } \omega=\omega_{p}
$$

Thus the perturbation shifts the maximum to the point where the derivative of the unperturbed power spectrum is compensated by the derivative of the perturbation $\delta P$. In a small frequency range around $\omega_{0}$ we can estimate the derivative of the unperturbed peak using the first-order term of the Taylor series expansion:

$$
\frac{d}{d \omega} P(\omega) \approx\left(\omega-\omega_{0}\right) \frac{d^{2} P}{d \omega^{2}}
$$

We have omitted the zero-order term of the Taylor series because the derivative of the unperturbed peak is equal to 0 at the peak maximum position, see eq A2. The second derivative of the unperturbed power spectrum in eq A7 must be estimated for the unperturbed peak frequency, $\omega=\omega_{0}$. The first derivatives in eq A6 are evaluated for $\omega=\omega_{p}$. However, considering a smooth behavior of the perturbation around $\omega_{0}$, we can use the value of the right-hand side in eq A6 for $\omega=\omega_{0}$. Combining eq A6 and A7 for $\omega=\omega_{p}$, we arrive at the sought relationship for the frequency shift caused by the perturbation, $\delta \omega \equiv \omega_{p}-\omega_{0}$ :

$$
\delta \omega \approx-\frac{d}{d \omega} \delta P\left(\frac{d^{2} P}{d \omega^{2}}\right)^{-1}
$$

This relationship may be applied for any type of perturbation provided that it is small enough for the approximation used in eq A7 to be reasonable. The second derivative of the unperturbed peak may be found from well-known line shape expressions, such as Lorentzian or sinc function [29]. The derivative of the perturbation in the numerator of eq A8 may be found using the approximation for $\delta P(\omega)$ in eq A5. An immediate consequence of the expressions eq A5 and A8 is that the frequency shift is a linear function of the perturbation. In other words, the combined effect produced by a number of peaks may be estimated as a sum of shifts produced by each of them, in the limit of small perturbations.

\section{Acknowledgments}

The authors gratefully acknowledge Dr. M. V. Gorshkov for helpful discussions. They thank the National Cancer Institute under grant CA86340 and the U.S. Department of Energy, Office of Biological and Environmental Research, for support of portions of this research. Pacific Northwest National Laboratory is operated by Battelle Memorial Institute for the U.S. Department of Energy through contract DE-AC06-76RLO 1830. 


\section{References}

1. Yates, J. R., III.; McCormack, A. L.; Eng, J. Mining Genomes with MS. Anal. Chem. 1996, 68, 534A-540A.

2. Jensen, O. N.; Podtelejnikov, A. V.; Mann, M. Identification of the Components of Simple Protein Mixtures by High-Accuracy Peptide Mass Mapping and Database Searching. Anal. Chem. 1997, 69, 4741-4750.

3. McLafferty, F. W.; Fridriksson, E. K.; Horn, D. M.; Lewis, M. A.; Zubarev, R. A. Biochemistry-Biomolecule Mass Spectrometry. Science 1999, 284, 1289-1290.

4. Clauser, K. R.; Baker, P.; Burlingame, A. L. Role of Accurate Mass Measurement $( \pm 10 \mathrm{ppm})$ in Protein Identification Strategies Employing MS or MS/MS and Database Searching. Anal. Chem. 1999, 71, 2871-2882.

5. Conrads, T. P.; Anderson, G. A.; Veenstra, T. D.; Pasa-Tolic, L.; Smith, R. D. Utility of Accurate Mass Tags for Proteome-Wide Protein Identification. Anal. Chem. 2000, 72, 3349-3354.

6. Shen, Y.; Zhao, R.; Belov, M. E.; Conrads, T. P.; Anderson, G. A.; Tang, K.; Pasa-Tolic, L.; Veenstra, T. D.; Lipton, M. S.; Smith, R. D. Packed Capillary Reversed-Phase Liquid Chromatography with High-Performance Electrospray Ionization Fourier Transform Ion Cyclotron Resonance Mass Spectrometry for Proteomics. Anal. Chem. 2001, 73, 1766-1775.

7. Shen, Y.; Tolic, N.; Zhao, R.; Pasa-Tolic, L.; Li, L.; Berger, S. J.; Harkewicz, R.; Anderson, G. A.; Belov, M. E.; Smith, R. D. High-Throughput Proteomics Using High-Efficiency Multiple-Capillary Liquid Chromatography with On-line High Performance ESI FTICR Mass Spectrometry. Anal. Chem. 2001, 73, 3011-3021.

8. Bruce, J. E.; Anderson, G. A.; Wen, J.; Harkewicz, R.; Smith, R. D. High Mass-Measurement Accuracy and 100\% Sequence Coverage of Enzymatically Digested Bovine Serum Albumin from an ESI-FTICR Mass Spectrum. Anal.Chem. 1999, 71, 2595-2599.

9. Pasa-Tolic, L.; Jensen, P. K.; Anderson, G. A.; Lipton, M. S.; Peden, K. K.; Martinovic, S.; Tolic, N.; Bruce, J. E.; Smith, R. D. High Throughput Proteome-Wide Precision Measurements of Protein Expression Using Mass Spectrometry. J. Am. Chem. Soc. 1999, 121, 7949-7950.

10. Smith, R. D. Probing Proteomes-Seeing the Whole Picture? Nature Biotechnol. 2000, 18, 1041-1042.

11. Mann, M.; Hojrup, P.; Roepstroff, P. Use of Mass Spectrometric Molecular Weight Information to Identify Proteins in Sequence Databases. Biol. Mass. Spectrom. 1993, 22, 338-345.

12. James, P.; Quadroni, M.; Carafoli, E.; Gonnet, G. Protein Identification by Mass Profile Fingerprinting. Biochem. Biophys. Res. Comm. 1993, 195, 58-64.

13. Yates, J.R., III.; Speicher, S.; Griffin, P. R.; Hunkapiller, T. Peptide Mass Maps: A Highly Informative Approach to Protein Identification. Anal. Biochem. 1993, 214, 397-408.

14. Masselon, C.; Anderson, G. A.; Harkewicz, R.; Bruce, J. E.; Pasa-Tolic, L.; Smith, R. D. Accurate Mass Multiplexed Tandem Mass Spectrometry for High-Throughput Polypeptide Identification from Mixtures. Anal. Chem. 2000, 72(8), 19181924.

15. Li, L.; Masselon, C. D.; Anderson, G. A.; Pasa-Tolic, L.; Lee, S.; Shen, Y.; Zhao, R.; Lipton, M. S.; Conrads, T. P.; Tolic, N.; Smith, R. D. High-Throughput Peptide Identification from Protein Digests Using Data-Dependent Multiplexed Tandem FTICR Mass Spectrometry Coupled with Capillary Liquid Chromatography. Anal. Chem. 2001, 73(14), 3312-3322.

16. Chen, S.; Comisarow, M. Simple Physical Models for Coulomb-Induced Frequency Shifts and Coulomb-Induced Inhomogeneous Broadening for Like and Unlike Ions in Fourier Transform Ion Cyclotron Resonance Mass Spectrometry. Rapid Commun. Mass Spectrom. 1991, 5, 450-455.
17. Chen, S.; Comisarow, M. Modeling Coulomb Effects in Fourier-transform Ion Cyclotron Resonance Mass Spectrometry by Charged Disks and Charged Cylinders. Rapid Commun. Mass Spectrom. 1992, 6, 1-3.

18. Xiang, X. Z.; Grosshans, P. B.; Marshall, A.G. Image ChargeInduced Ion-Cyclotron Orbital Frequency-Shift for Orthorhombic and Cylindrical FT-ICR Ion Traps. Int. J. Mass Spectrom. Ion Processes 1993, 125, 33-43.

19. Gorshkov, M. V.; Marshall, A. G.; Nikolaev, E. N. Analysis and Elimination of Systematic Errors Originating from Coulomb Mutual Interaction and Image Charge in Fourier Transform Ion Cyclotron Resonance Precise Mass Difference Measurements. J. Am. Soc. Mass Spectrom. 1993, 4, 855-868.

20. Pasa-Tolic, L.; Huang, Y.; Guan, S.; Kim, H. S.; Marshall, A. G. Ultrahigh-Resolution MALDI FTICR Mass Spectra of Peptides. J. Mass Spectrom. 1995, 30, 825-833.

21. Mitchell, D. W.; Smith, R. D. Cyclotron Motion of Two Coulombically Interacting Ion Clouds with Implications to Fourier-Transform Ion Cyclotron Resonance Mass Spectrometry. Phys. Rev. E 1995, 52, 4366-4386.

22. Mitchell, D. W.; Smith, R. D. Prediction of a Space Charge Induced Upper Molecular Mass Limit Towards Achieving Unit Mass Resolution in Fourier Transform Ion Cyclotron Resonance Mass Spectrometry. J. Mass Spectrom. 1996, 31, 771-790.

23. Naito, Y.; Inoue, M. Collective Motion of Ions in an Ion Trap for Fourier Transform Ion Cyclotron Resonance Mass Spectrometry. Int. J. Mass Spectrom. Ion Processes 1996, 157/158, 85-96.

24. Marshall, A. G.; Comisarow, M. B.; Parisod, G. Relaxation and Spectral Line Shape in Fourier Transform Ion Cyclotron Resonance Spectroscopy. J. Chem. Phys. 1979, 71, 4434-4444.

25. Marshall, A. G.; Hendrickson, C. L.; Jackson, G. S. Fourier Transform Ion Cyclotron Resonance Mass Spectrometry: A Primer. Mass Spectrom. Rev. 1998, 17, 1-35.

26. Posener, D. W. Precision in Measuring Resonance Spectra. J. Magnetic Reson. 1974, 14, 121-128.

27. Chen, L.; Cottrell, C. E.; Marshall, A. G. Effect of Signal-toNoise Ratio and Number of Data Points Upon Precision in Measurement of Peak Amplitude, Position, and Width in Fourier-Transform Spectrometry. Chemometrics Intel. Lab. Syst. 1986, 1, 51-58.

28. Liang, Z.; Marshall, A. G. Time-Domain (Interferogram) and Frequency-Domain (Absorption-Mode and Magnitude-Mode) Noise and Precision in Fourier Transform Mass Spectrometry. Applied Spectros. 1990, 44, 766-775.

29. Marshall, A. G.; Verdun, F. R. Fourier Transforms in NMR, Optical, and Mass Spectrometry; Elsevier: Amsterdam 1990; pp $1-450$.

30. Craig, E. C.; Marshall, A. G. Automated Phase Correction Of FT NMR-Spectra by Means of Phase Measurement Based on Dispersion versus Absorption Relation (DISPA). J. Magnetic Reson. 1988, 76, 458-475.

31. Craig, E. C.; Santos, I.; Marshall, A. G. Dispersion versus Absorption (DISPA) Method for Automatic Phasing of Fourier Transform Ion Cyclotron Resonance Mass Spectra. Rapid Commun. Mass Spectrom. 1987, 1, 33-37.

32. Vining, B. A.; Bossio, R. E.; Marshall, A. G. Phase Correction for Collision Model Analysis and Enhanced Resolving Power of Fourier Transform Ion Cyclotron Resonance Mass Spectra. Anal. Chem. 1999, 71, 460-467.

33. Wang, M.; Marshall, A. G. Mass Shifts Induced by Negative Frequency Peaks in Linearly Polarized Fourier-Transform Ion-Cyclotron Resonance Signals. Int. J. Mass Spectrom. Ion Processes 1988, 86, 31-51.

34. Lee, J. P.; Comisarow, M. B. The Phase Dependence of Magnitude Spectra. J. Magnetic Reson. 1987, 72, 139-142. 
35. Lee, J. P.; Chow, K. H.; Comisarow, M. B. Anomalous Intensities of Apodized and Unapodized Magnitude Spectra. Anal. Chem. 1988, 60, 2212-2218.

36. Chow, K. H.; Comisarow, M. B. Frequency (Mass) Errors and Phase Dependence in Magnitude-Mode Apodized Fourier Transform-Ion Cyclotron Resonance Spectra. Int. J. Mass Spectrom. Ion Processes 1989, 89, 187-203.

37. Aarstol, M.; Comisarow, M. B. Apodization of FT-ICR Spectra. Int. J. Mass Spectrom. Ion Processes 1987, 76, 287-297.

38. Comisarow, M. B.; Marshall, A. G. Theory of Fourier Transform Ion Cyclotron Resonance Mass Spectrometry. I. Fundamental Equations and Low-Pressure Line Shape. J. Chem. Phys. 1976, 64, 110-119.

39. Rade, L.; Westergren, B. Mathematics Handbook for Science and Engineering, 4th ed.; Springer: New York, 1998; p 316.

40. Udseth, H. R.; Gorshkov, M. V.; Belov, M. L.; Pasa-Tolic, L.; Bruce, J. E.; Masselon, C. D.; Harkewicz, R.; Anderson, G. A.; Smith, R. D. Proceedings of the 37th ASMS Conference on Mass Spectrometry And Allied Topics; 1999; Dallas, TX. June.

41. Kim, T.; Tolmachev, A. V.; Harkewicz, R.; Anderson, G. A.; Udseth, H. R.; Futrell, J. H.; Smith, R. D. Design and Implementation of a New Electrodynamic Ion Funnel. Anal. Chem. 2000, 72, 2247-2255.

42. Tolmachev, A. V.; Kim, T.; Udseth, H. R.; Smith, R. D.; Bailey, T. H.; Futrell, J. H. Simulation-Based Optimization of the
Electrodynamic Ion Funnel for High Sensitivity Electrospray Ionization Mass Spectrometry. Int. J. Mass Spectrom. 2000, 203, 31-47.

43. Belov, M. E.; Gorshkov, M. V.; Udseth, H. R.; Anderson, G. A.; Tolmachev, A. V.; Prior, D. C.; Harkewicz, R.; Smith, R. D. Initial Implementation of an Electrodynamic Ion Funnel with Fourier Transform Ion Cyclotron Resonance Mass Spectrometry. J. Am. Soc. Mass Spectrom. 2000, 11, 19-23.

44. Marshall, A. G. Theory for Ion Cyclotron Resonance Absorption Line Shapes. J. Chem. Phys. 1971, 55, 1343-1354.

45. Guan, S.; Li, G. Z.; Marshall, A. G. Effect of Ion-Neutral Collision Mechanism on the Traped-Ion Equation of Motion: A New Mass Spectral Line Shape for High-Mass Trapped ions. Int. J. Mass Spectrom. Ion Processes 1997, 167/168, 185-193.

46. Bruce, J. E.; Anderson, G. A.; Brands, M. D.; Pasa-Tolic, L.; Smith, R. D. Obtaining More Accurate Fourier Transform Ion Cyclotron Resonance Mass Measurements Without Internal Standards Using Multiply Charged Ions. J. Am. Soc. Mass Spectrom. 2000, 11, 416-421.

47. Guan, S.; Wahl, M. C.; Marshall, A. G. Elimination of Frequency Drift from Fourier Transform Ion Cyclotron Resonance Mass Spectra by Digital Quadrature Heterodyning: Ultrahigh Mass Resolving Power for Laser-Desorbed Molecules. Anal. Chem. 1993, 65, 3647-3653. 